VOL. 19 (1978), 205-216.

\title{
Path decompositions of digraphs
}

\section{Issam Abdul-Kader}

Let $G=(X, U)$ be a digraph of order $n . P(G)$ denotes the minimal cardinal of a path-partition of the arcs of $G$. Oystein Ore, Theory of graphs (Amer. Math. Soc., Providence, Rhode Island, 1962) has proved that $P(G) \geq \sum_{x \in X_{G}^{+}}\left(d_{G}^{+}(x)-d_{G}^{-}(x)\right)$, where $X_{G}^{+}=\left\{x \in X \mid d_{G}^{+}(x)>d_{G}(x)\right\}$. We say. that $G$ satisfies $Q$ if the preceeding inequality is an equality.

We give some properties of the digraphs satisfying $Q$, and in particular the case where $G$ is strongly connected. Then we prove that $P(G) \leq\left[n^{2} / 4\right]-2$, and that this result is the best possible. Next we exhibit the existence of digraphs with circuits such that $P(G)=\left[n^{2} / 4\right]$.

Finally we prove that if $G$ is a strongly connected digraph of order $n$ which satisfies $Q$, then there exists a strongly connected digraph $H$ of order $n+1$ having $G$ as a sub-digraph and satisfying $Q$, too.

\section{Introduction}

1.1. The notations are those of Berge [5].

A digraph $G=(X, U)$ is a non-empty finite set $X$ (the vertices), together with a finite family $U$ of ordered pairs of vertices (the arcs). A simple digraph is a digraph without parallel arcs and loops.

In this paper we only consider simple digraphs. The digraph obtained Received 8 August 1978. Communicated by Michel Chein. 
from $G$ by deleting a vertex $x \in X$ and its adjacent arcs will be denoted by $G-x$.

We denote by $\left(x_{1}, x_{2}, \ldots, x_{k}\right)$ (respectively $\left(x_{1}, x_{2}, \ldots, x_{k}, x_{1}\right)$ ) the elementary path (respectively the circuit) containing the $k$ distinct vertices $x_{1}, \ldots, x_{k}$. Let $R$ be a family of elementary paths of $G$. If each arc of $G$ lies on exactly one element of $R$ then $R$ is a pathpartition of $G$. We denote by $P(G)$ the minimal cardinality of a pathpartition of a digraph $G$.

From now on we denote

$$
\begin{aligned}
\Gamma_{G}^{+}(x) & =\{y \in X \mid(x, y) \in U\}, \quad\left(d_{G}^{+}(x)=\left|\Gamma_{G}^{+}(x)\right|\right), \\
\Gamma_{G}^{-}(x) & =\{y \in X \mid(y, x) \in U\}, \quad\left(d_{G}(x)=\left|\Gamma_{G}^{-}(x)\right|\right), \\
X_{G}^{+} & =\left\{x \in X \mid d_{G}^{+}(x)>d_{G}^{-}(x)\right\}, \\
X_{G}^{0} & =\left\{x \in X \mid d_{G}^{+}(x)=d_{G}(x)\right\}, \\
X_{G}^{-} & =X-\left(X_{G}^{+} \cup X_{G}^{0}\right\} .
\end{aligned}
$$

From Ore [9], we have

$$
P(G) \geq \sum_{x \in X_{G}^{+}}\left(d_{G}^{+}(x)-d_{G}(x)\right)
$$

Alspach and Pullman [4], have conjectured that for any simple digraph $G$ or order $n, P(G) \leq\left[n^{2} / 4\right]$.

O'Brien [8] proved this conjecture. For a further detailed study of the index $P(G)$, we refer also to Chaty, Chein ([6], [7]).

DEFINITION 1.2. Let $G=(X, U)$ be a digraph of order $n$; if $P(G)=\sum_{x \in X_{G}^{+}}\left(d_{G}^{+}(x)-d_{G}(x)\right)$ we say $G$ has the property $Q$. In the following, we denote by $e(G)$ the sum $e(G)=\sum_{x \in X_{G}^{+}}\left(d_{G}^{+}(x)-d_{G}(x)\right)$. 


\section{Results}

LEMMA 2.1. Let $G=(X, U)$ be a digraph of order $n$ and $v \in X_{G}^{+}$. If $G$ satisfies the following conditions,

(i) $\bar{d}_{G}(v)=0$,

(ii) $P(G-v)=e(G-v)$ (that is $G-v$ has the property $Q$ ), then $G$ has the property $Q$.

Proof. $X_{G-v}^{+}=\left(X_{G}^{+}\{v\}\right) \cup\left(X_{G}^{0} \cap \Gamma_{G}^{+}(v)\right\}$, and $P(G-v)=e(G-v)$. If $x \in X_{G-v}^{+}$, then $d_{G-v}^{+}(x)=d_{G}^{+}(x)$ and $d_{G-v}^{-}(x)=d_{G}^{-}(x)-1$. Moreover, for $x \in X_{G-v}^{+}-\left(X_{G-v}^{+} \cap \Gamma_{G}^{+}(v)\right)$, we have

$$
d_{G-v}^{+}(x)=d_{G}^{+}(x) \text { and } d_{G-v}(x)=d_{G}(x) \text {. }
$$

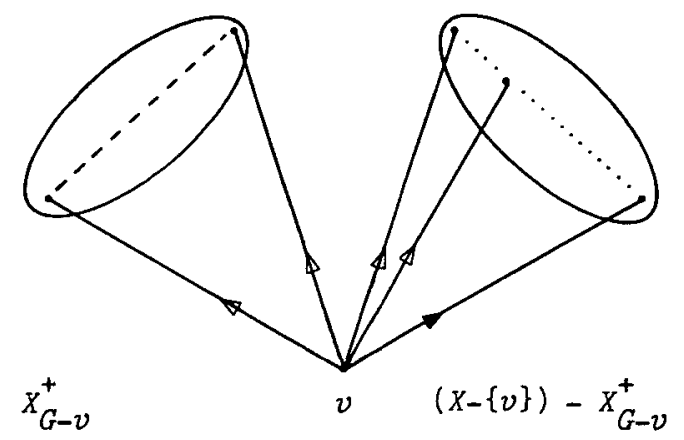

But in $G-v$, through each vertex $x \in X_{G-v}^{+} \cap \Gamma_{G}^{+}(v)$ there pass $d_{G-v}(x)-d_{G-v}(x)=\left(d_{G}^{+}(x)-d_{G}(x)\right)+1$ elementary paths of origin $x$, which belong to a path-partition $R$ of the arcs of the digraph, the cardinal of $R$ being $P(G-v)$. Among those paths of origin $x$, consider the path $\lambda=(x, \ldots)$. Since $(v, x) \in U$, the path $\lambda$ allows the construction in $G$ of the path $\mu=(v, x, \ldots)$ of origin $v$. Thus the number of paths of origin $x$ in $G$ becomes $\left(d_{G}^{+}(x)-d_{G}(x)\right)$. Moreover, for each $x \in \Gamma_{G}^{+}(v)-\left(X_{G-v}^{+} \cap \Gamma_{G}^{+}(v)\right)$, we construct the path $(v, x)$ of origin $v$ in $G$. Let $R^{\prime}$ be the set of elementary paths obtained from $R$ by cancelling those paths $\lambda$ which have been used to define the path $\mu$ of origin $v$ in $G$. Let $T$ be the following set of elementary paths: 
$T=R^{\prime} \cup\left\{\mu=(v, x, \ldots) \mid x \in X_{G-v}^{+} \cap \Gamma_{G}^{+}(v)\right\}$

$$
u\left\{(v, x) \mid x \in \Gamma_{G}^{+}(v)-\left(X_{G-v}^{+} \cap \Gamma_{G}^{+}(v)\right)\right\} .
$$

It is obvious that the set $T$ partitions the arcs of $G$, and we have

$$
|T| \leq e(G) \leq P(G) \text {. }
$$

Therefore $P(G)=e(G)$.

From the preceding lemma, we deduce the following theorem.

THEOREM 2.2 (Ore [9]). Let $G=(X, U)$ be a digraph without circuit; then

$$
P(G)=e(G) \text {. }
$$

COROLLARY 1 (Alspach and Pullman [4]). If $T T_{n}$ is the transitive tournament of order $n$ we have

$$
P\left(T T_{n}\right)=\left[n^{2} / 4\right]
$$

REMARKS. (1) If we replace the condition (i) of the lemme by the condition $\left(i^{\prime}\right), d_{G}^{+}(v)=0$, we get a similar result. Moreover, the preceding lemma allows us to construct from a digraph of order $(n-1)$ satisfying $Q$, another digraph of order $n$ still satisfying $Q$.

(2) By that lemma, we can define an algorithm which allows the construction of a path-partition of a digraph without circuit.

The following lemma is due to Alspach, Mason, Pullman [3].

LEMMA 2.3. Let $G=(X, U)$ be a digraph of order $n$ satisfying $Q$ and $(x, y)$ an are of $G$ such that $x \in X-X_{G}^{+}$and $y \in X_{G}^{+} \cup X_{G}^{0}$. If $H$ is the digraph obtained from $G$ by reversing the are $(x, y)$, then $H$ satisfies $Q$ and $P(H)=P(G)+2=e(H)$.

THEOREM 2.4. Consider a strongly connected digraph $G=(X, U)$ satisfying $P(G)=e(G)$. Then we have

$$
P(G) \leq\left[n^{2} / 4\right]-2
$$

Proof. Suppose that $P(G) \geq\left[n^{2} / 4\right]-1$. Since $G$ is strongly connected, there exist $x \in X-X_{G}^{+}$and $y \in X_{G}^{+}$such that $(x, y) \in U$. 
Denote by $G_{1}$ the digraph obtained from $G$ by reversing the arc $(x, y)$. By Lemma 2.3, $G_{1}$ satisfies $Q$ and we have

$$
P\left(G_{1}\right)=P(G)+2 \geq\left[n^{2} / 4\right]+1
$$

which is a contradiction to the fact that $P\left(G_{1}\right) \leq\left[n^{2} / 4\right]$. Thus we necessarily have $P(G) \leq\left[n^{2} / 4\right]-2$.

We show that the result of Theorem 2.4 is the best possible.

REMARKS. (1) Let $T_{n}=(X, U)$ be a tournament of order $n$. It is easy to verify that $P\left(T_{n}\right) \geq[(n+1) / 2]$; therefore

$$
[(n+1) / 2] \leq P\left(T_{n}\right) \leq\left[n^{2} / 4\right] \text {. }
$$

(2) Let $A_{n}=(X, U)$ be the strongly connected c-minimal tournament ${ }^{1}$ of order $n$ (that is $A_{n}=(X, U)$ admits exactly $((n-1)(n-2)) / 2$ elementary circuits). Let us study some particular cases. Case $n=4$.

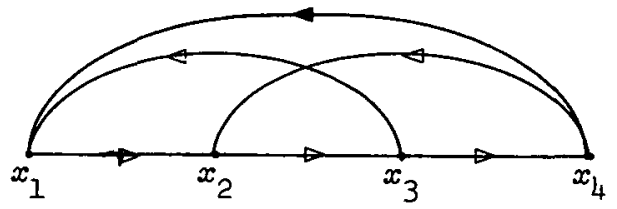

Let $x_{1}, x_{2}, x_{3}, x_{4}$ be the canonical indexation of the vertices of $A_{4}$. We have

$$
P\left(A_{4}\right) \geq e\left(A_{4}\right)=2 \text {. }
$$

But the set $\left\{\left(x_{4}, x_{2}, x_{3}, x_{1}\right),\left(x_{3}, x_{4}, x_{1}, x_{2}\right)\right\}$ of elementary paths forms a partition of the arcs of $A_{4}$. Therefore $P\left(A_{4}\right)=\left[n^{2} / 4\right]-2=2$.

1 A complete study of strongly connected c-minimal tournaments will be found in Abdul-Kader [1]. 
Case $n=5$.

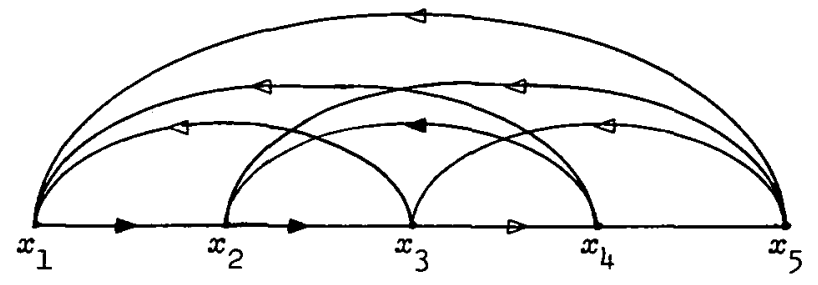

Let $x_{1}, x_{2}, x_{3}, x_{4}, x_{5}$ be the canonical indexation of the vertices of $A_{5}$. We have

$$
P\left(A_{5}\right) \geq e\left(A_{5}\right)=4 \text {. }
$$

But the set

$$
\left\{\left(x_{5}, x_{2}, x_{3}, x_{1}\right),\left(x_{5}, x_{3}, x_{4}, x_{2}\right),\left(x_{4}, x_{1}, x_{2}\right),\left(x_{4}, x_{5}, x_{1}\right)\right\}
$$

of elementary paths forms a partition of the arcs of $A_{5}$. Therefore

$$
P\left(A_{5}\right)=\left[n^{2} / 4\right]-2=4=e\left(A_{5}\right) \text {. }
$$

THEOREM 2.5. Let $A_{n}=(X, U)$ be the strongly connected c-minimal toumament of order $n \geq 5$; then

$$
P\left(A_{n}\right)=\left[n^{2} / 4\right]-2=e\left(A_{n}\right) .
$$

Proof. We prove the theorem by induction on $n$. The theorem is already true for $n=4,5$. Suppose it is true for $A_{n-1}$; we prove it for $A_{n} \quad(n \geq 6)$.

Let $x_{1}, x_{2}, \ldots, x_{n}$ be the canonical indexation of the vertices of $A_{n}$.

(1) Let $G$ be the digraph obtained from $A_{n-1}$ by adding the vertex $y$ and the ares $\left(y, x_{i}\right)$ for all $i=1, \ldots, n-2$. By the induction hypothesis $A_{n-1}$ satisfies $Q$, which implies that the digraph $G$ satisfies $Q$ (see Lemma 2.I).

(2) Let $G_{1}$ be the digraph obtained from $G$ by adding the arc 
$\left(x_{n-1}, y\right)$. But $d_{G_{1}}\left(x_{n-1}\right)=1$ and $n>5$; hence there exists at least one path $\lambda$ of origin $y$ which does not end up at the point $x_{n-1}$. From this path $\lambda$ we can construct a path of origin $x_{n-1}$ in $G_{1}$; therefore $P\left(G_{1}\right)=e\left(G_{1}\right) \quad\left(G_{1}\right.$ isomorphic to $\left.A_{n}\right)$.

In $A_{n}$ we have (see Abdul-Kader [1]),

$$
X_{A}^{+}=\left\{x_{i} \mid i=[n / 2]+1, \ldots, n\right\},
$$

$$
d_{A_{n}}^{+}\left(x_{i}\right)-d_{A_{n}}\left(x_{i}\right)=2(i-1)-(n-1) \text { for all } i=[n / 2]+1, \ldots, n-1 \text {, }
$$$$
d_{A_{n}}^{+}\left(x_{n}\right)-d_{A_{n}}\left(x_{n}\right)=n-3 \text {. }
$$

Therefore

$$
\begin{aligned}
P\left(A_{n}\right) & =\sum_{i=[n / 2]+1}^{n-1} 2(i-1)-(n-1)+(n-3) \\
& =e\left(A_{n}\right)=\left[n^{2} / 4\right]-2 .
\end{aligned}
$$

The following corollary proves the existence, by exhibiting them, of digraphs $G$ with circuits satisfying $P(G)=\left[n^{2} / 4\right]$.

We denote by $T T_{n}$ the transitive tournament of order $n$.

COROLLARY 1. There exist toumaments $T_{n}$ which are not isomorphic to $T T_{n}$ and such that

$$
P\left(T_{n}\right)=\left[n^{2} / 4\right]
$$

Proof. Let $A_{n}=(X, U)$ be the tournament strongly connected and c-minimal of order $n$. Let $x_{1}, x_{2}, \ldots, x_{n}$ be the canonical indexation of the vertices of $A_{n}$.

First Case: $n=2 k$.

In $A_{2 k}$ we have: if $d_{A_{2 k}^{+}}\left(x_{k}\right)=k-1$ and $d_{A_{2 k}}\left(x_{k}\right)>k-1$, then $d_{A_{2 k}}^{+}\left(x_{k}\right)-d_{A_{2 k}}\left(x_{k}\right)<0$; moreover $d_{A_{2 k}}^{+}\left(x_{k+1} j-d_{A_{2 k}}\left(x_{k+1}\right)>0\right.$. We 
denote by $T_{n}$ the tournament of order $n$ obtained from $A_{n}$ by reversing the arc $\left(x_{k}, x_{k+1}\right)$.

By Lemma 2.3, the tournament $T_{n}$ satisfies $Q$ and we have

$$
P\left(T_{n}\right)=P\left(A_{n}\right)+2=\left[n^{2} / 4\right]=e\left(T_{n}\right) .
$$

Second Case: $n=2 k+1$.

We have

$$
\begin{array}{r}
d_{A_{n}}^{+}\left(x_{k}\right)-d_{A_{n}}\left(x_{k}\right)<0, \\
d_{A_{n}}^{+}\left(x_{k+1}\right)-d_{A_{n}}\left(x_{k+1}\right)=0,
\end{array}
$$

and

$$
d_{A_{n}}^{+}\left(x_{k+2}\right)-d_{A}\left(x_{k+2}\right)>0
$$

Let $T_{n}$ be the tournament of order $n$ obtained from $A_{n}$ by reversing the arc $\left(x_{k}, x_{k+1}\right)$; by Lemma 2.3 , the tournament satisfies $Q$ and $P\left(T_{n}\right)=P\left(A_{n}\right)+2-\left[n^{2} / 4\right]=e\left(T_{n}\right)$. Similarly the tournament of order $n$ obtained from $A_{n}$ by reversing the arc $\left(x_{k+1}, x_{k+2}\right)$ satisfies $Q$ and $P\left(T_{n}\right)=P\left(A_{n}\right)+2=\left[n^{2} / 4\right]=e\left(T_{n}\right)$. This proves our result.

By Abdul-Kader [2], if $T_{n}$ is a tournament having a unique hamiltonian circuit, we have

(1) $P\left(T_{n}\right) \leq\left[n^{2} / 4\right]-2$;

(2) this result is the best possible, that is, there exist tournaments having a unique hamiltonian circuit, which are not isomorphic to $A_{n}$, and which satisfy the equation $P\left(T_{n}\right)=\left[n^{2} / 4\right]-2 ;$

(3) $T_{n}$ does not satisfy the property $Q$, in general.

THEOREM 2.6. Let $G=(X, U)$ be a strongly connected digraph of 
order $n$ satisfying $Q$; then there exists a strongly connected digraph $H$ of order $n+1$, satisfying $Q$ and having $G$ as a sub-digraph.

Proof. We have $X_{G}^{+} \neq \emptyset$.

First Case: $\left|X_{G}^{-}\right| \geq\left|X_{G}^{+}\right|$.

Let $B_{1}, B_{2}$ be a partition of $X_{G}^{-}$such that $\left|B_{1}\right|=\left|X_{G}^{+}\right|$. Let $x_{0} \vDash X$ and $B$ be the digraph generated by $X \cup\left\{x_{0}\right\}$ such that

(1) $G \subset H$,

(2) for all $x \in X_{G}^{+} \cup B_{1}$, we consider the arcs $\left(x, x_{0}\right)$ if $x \in X_{G}^{+}$and $\left(x_{0}, x\right)$ if not, as being arcs of $H$. We have then $X_{H}^{+}=X_{G}^{+}$. Moreover, $d_{H}^{+}(x)=d_{G}^{+}(x)+1$ and $\widetilde{d_{H}}(x)=\sigma_{G}(x)$ for all $x \in X_{H}^{+}$; then

$$
P(H) \geq e(H)=e(G)+\left|X_{G}^{+}\right| \text {. }
$$

If $R$ is a path-partition of $G$ such that $|R|=P(G)$, then the set $R_{1}=R \cup\left\{\left(x_{i}, x_{0}, b_{i}\right) \mid x_{i} \in X_{G}^{+}, b_{i} \in B_{1}\right\}$ is a path-partition of the arcs of $H$ and $\left|R_{1}\right|=P(G)+\left|X_{H}^{+}\right|$. One verifies easily that the digraph $H$ is strongly connected; therefore

$$
P(H)=e(H) \text {. }
$$

Second Case: $\left|X_{G}^{-}\right|<\left|X_{G}^{+}\right|$.

Let $C_{1}, C_{2}$ be a partition of $X_{G}^{+}$such that $\left|C_{1}\right|=\left|X_{G}^{-}\right|$, and let $H$ denote the digraph generated by $X \cup\left\{x_{0}\right\}$ and satisfying

(1) $G \subset H$,

(2) for all $x \in C_{1} \cup X_{G}^{-}$, we consider the arcs $\left(x, x_{0}\right)$ if $x \in C_{1}$ and $\left(x_{0}, x\right)$ if $x \in X_{G}^{-}$, as being arcs of $H$.

We have the relations

$$
\begin{aligned}
& d_{H}^{+}(x)=d_{G}^{+}(x)+1, d_{H}(x)=\sigma_{G}(x) \text { for all } x \in C_{1}, \\
& d_{H}^{+}(x)=d_{G}^{+}(x) \quad, d_{H}(x)=\sigma_{G}(x) \text { for all } x \in c_{2} .
\end{aligned}
$$


Then

$$
P(H) \geq e(H)=e(G)+\left|C_{1}\right| .
$$

Moreover the set $R_{1}=. R \quad\left\{\left(x_{i}, x_{0}, y_{i}\right\} \mid x_{i} \in C_{1}, y_{i} \in X_{G}^{-}\right\}$ partitions the arcs of $H$ and $\left|R_{1}\right|=|R|+\left|C_{1}\right|=P(G)+\left|C_{1}\right|$. Therefore $P(H)=e(H)$. As before, one easily verifies that $H$ is strongly connected.

REMARK. This last theorem constitutes a procedure of extension permitting the construction, from a class of strongly connected digraphs satisfying $Q$, another class of strongly connected digraphs satisfying $Q$ too.

EXAMPLES. Consider a strongly connected digraph $G$ of order $n$ satisfying $P(G)=e(G)$. We study two cases.

(1) $n=5$.

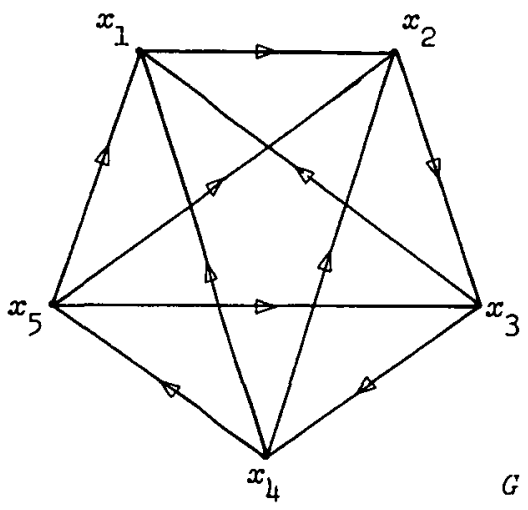

By Theorem 2.6, we have 


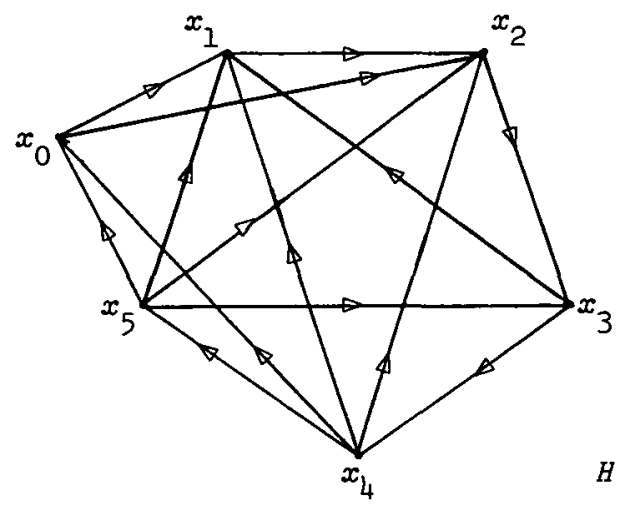

(2) $n=6$.

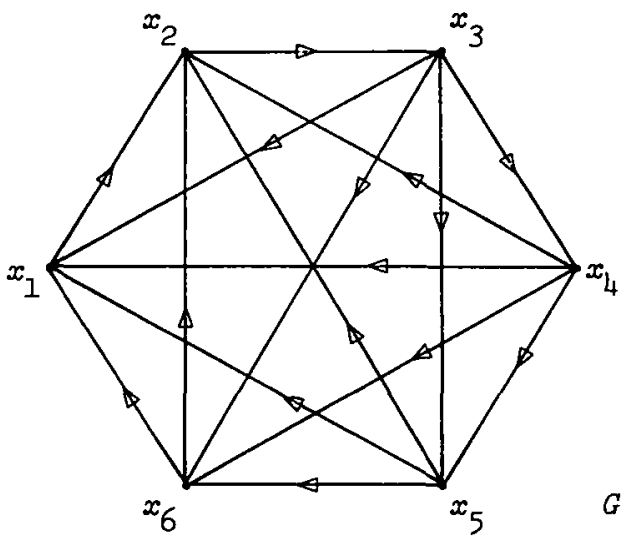

By Theorem 2.6 we have

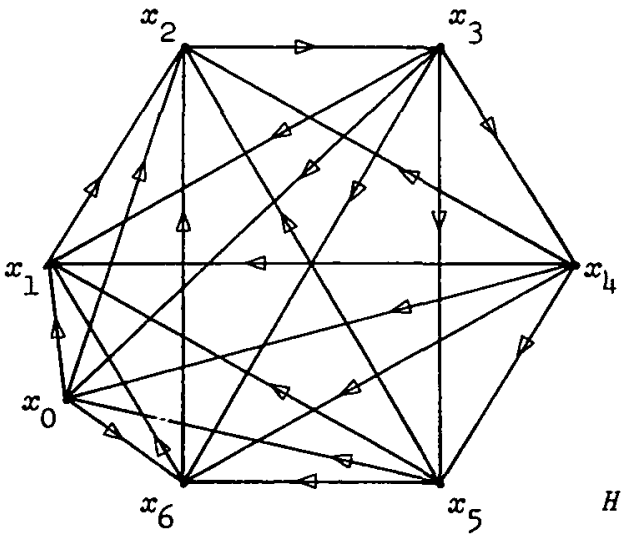




\section{References}

[1] Issam Abdul-Kader, "Sur des graphes ayant un circuit hamiltonien unique et sur des invariants des cheminements dans les graphes orientés" (Thèses de Doctorat d'Etat lere, Université Pierre et Marie Curie, Paris, 1977).

[2] Issam Abdul-Kader, "Indice de partition en chemins des arcs des tournois ayant un seul circuit hamiltonien", Problèmes combinatoires et théorie des grophes, orsay, Juillet, 1976, 1-2 (Colloques Internationaux du Centre National de la Recherche Scientifique, 260. Centre National de la Recherche Scientifique, Paris, 1978).

[3] Brian Alspach, David W. Mason, Norman J. Pullman, "Path numbers of tournaments", preprint.

[4] Brian R. Alspach and Norman J. Pullman, "Path decompositions of digraphs", BuZZ. Austral. Math. Soc. 10 (1974), 421-427.

[5] C. Berge, Graphes et hypergraphes (Dunod Université, 604. Dunod, Paris, 1970).

[6] G. Chaty et M. Chein, "Invariants liés aux chemins dans les graphes sans circuits", Colloquio Internazionale sulle Teorie Combinatorie con la collaborazione della American Mathematical Society, Roma, 1973, Tomo I, 287-308 (Atti dei Convegni Lincei, 17. Academia Nazionale dei Lincei, Roma, 1976).

[7] G. Chaty, M. Chein, "Path-number of k-graphs and symmetric digraphs", Proceedings of the Seventh Southeastern Conference on Combinatorics, Graph Theory, and Computing, 203-216 (Louisana State University, Baton Rouge, 1976. Congressus Numerantium, 17. Utilitas Mathematica, Winnipeg, 1976).

[8] Richard C. O'Brien, "An upper bound on the path number of a digraph", J. Combinatorial Theory Ser. B 22 (1977), 168-174.

[9] Oystein Ore, Theory of graphs (American Mathematical Society Colloquium Publications, 38. American Mathematical Society, Providence, Rhode Island, 1962).

Faculty of Science, Lebanese University, Beirut, Lebanon. 